\title{
Cloning and sequencing of two Candida parapsilosis genes encoding acid proteases
}

\author{
Pierre A. De Viragh, ${ }^{1}$ Dominique Sanglard,${ }^{2}$ Giuseppe Togni, ${ }^{1}$ Rocco Falchetto ${ }^{3}$ \\ and MiCHEL MONOD ${ }^{1 *}$ \\ ${ }^{1}$ Laboratoire de Mycologie, Service de Dermatologie, Centre Hospitalier Universitaire Vaudois, 1011 Lausanne, \\ Switzerland \\ ${ }^{2}$ Institut für Biotechnologie ETH-Hönggerberg, 8093 Zürich, Switzerland \\ ${ }^{3}$ Laboratorium für Biochemie, ETH-Zentrum, 8092 Zürich, Switzerland
}

(Received 19 June 1992; revised 4 September 1992; accepted 29 September 1992)

\begin{abstract}
Candida parapsilosis secretes an inducible acid protease (ACP) when cultivated in the presence of bovine serum albumin as the sole nitrogen source. In order to clone the ACP gene $(A C P)$ of $C$. parapsilosis, a genomic library was screened with $C$. tropicalis $A C P$ as the probe. Two different $\mathrm{ORFs}, A C P R$ and $A C P L$, were found to hybridize with the $C$. tropicalis $A C P$. $A C P R$ contained a DNA sequence in agreement with the $\mathrm{N}$-terminal amino acid sequence of $C$. parapsilosis ACP isolated from culture supernatants. $A C P R$ was shown to be expressed and functional in a $C$. tropicalis acid protease mutant ( $a c p)$ and with SDS-PAGE the protein product showed the same mobility as the ACP secreted by $C$. parapsilosis. These results imply that $A C P R$ encodes the $C$. parapsilosis ACP. The deduced amino acid sequence of $A C P R$ is similar to the amino acid sequence of proteases of the pepsin family. As in the case of the $C$. tropicalis and $C$. albicans $A C P$, the $5^{\prime}$ extremity of $A C P R$ revealed a propeptide containing two Lys-Arg amino acid pairs that have been identified as peptidase processing sites in several yeast-secreted peptides and protein precursors. As judged from the deduced amino acid sequences, the $A C P L$ product would be similar to that of $A C P R$; however, a protein corresponding to $A C P L$ was not found in supernatants from $C$. parapsilosis liquid cultures. In addition, $A C P L$ did not complement the $C$, tropicalis acp mutant. We conclude that $A C P L$ is a pseudogene or serves an as yet unidentified function.
\end{abstract}

\section{Introduction}

Candida albicans, C. tropicalis and C. parapsilosis, three opportunistic yeast species of medical interest, secrete acid proteases (ACP) in vitro, when proteins are the sole nitrogen source in the medium (Rüchel et al., 1983). Individual ACPs from the three species have been characterized at the protein level (Remold et al., 1968; MacDonald \& Odds, 1980; Rüchel, 1981; Negi et al., 1984; Rüchel et al., 1986; Shimizu et al., 1987; Ray \&

*Author for correspondence. Tel 41213142823 ; fax 412131428 25 .

Abbreviations: ACP, extracellular acid protease; $A C P$, extracellular acid protease gene; PAS, periodic acid-Schiff reagent. $A C P R$ and $A C P L$ designate the two $C$. parapsilosis open reading frames described in this work.

The nucleotide sequence data reported in this paper have been submitted to GenBank and have been assigned the accession numbers Z11918 ( $A C P L)$ and Z11919 (ACPR).
Payne, 1990). C. tropicalis and C. albicans ACP are $41-43 \mathrm{kDa}$ glycoproteins with a pI of $4 \cdot 5$. C. parapsilosis ACP has a lower apparent molecular mass of $33 \mathrm{kDa}$ and a higher pI of 5.7 (Rüchel et al., 1986). Candida acid proteases have optimal activity between $\mathrm{pH} 4.0$ and 4.5 and are inactivated below $\mathrm{pH} 2.5$ and above $\mathrm{pH} 6.0$. These enzymes have a broad substrate specificity including keratin, denaturated collagen, haemoglobin and bovine serum albumin. They are inactivated by pepstatin and consequently belong to the pepsin family. Results of immunological studies suggest the existence of common and specific domains among the acid proteases of the three species (Rüchel et al., 1986). Recently, the genes coding for ACP of C. albicans and C. tropicalis were isolated and sequenced (Hube et al., 1991; Togni et al., 1991). The protein sequences deduced from the two cloned genes both include regions of homology to the active sites of proteases of the pepsin family.

C. parapsilosis like other Candida species, causes fungemia, but is more prevalent in patients with solid tumours (Horn et al., 1985; Komshian et al., 1989). 
Studies on Candida ACP are expected to elucidate the pathogenicity of these organisms. For $C$. albicans and $C$. tropicalis, proteolytic activity may be associated with tissue invasion, since ACP is expressed at the surface of the fungus during invasion (Rüchel et al., 1991) and preliminary studies for $C$. albicans have shown a correlation between proteolytic activities and virulence (MacDonald \& Odds, 1983; Kwon-Chung et al., 1985; Ross et al., 1990). Although C. parapsilosis secretes a large amount of ACP in vitro, this species is much less invasive than C. albicans and C. tropicalis (Bistoni et al., 1984). This discrepancy could be related to the expression of ACP in macrophages after phagocytosis of yeast since $C$. albicans and $C$. tropicalis, but not $C$. parapsilosis, express ACP on the surface of yeast cells in macrophages after phagocytosis (Borg \& Rüchel, 1990).

We report the cloning and the nucleotide sequence of two tandemly arranged $C$. parapsilosis acid protease genes, $A C P R$ and $A C P L$. $A C P R$ encodes the $C$. parapsilosis ACP found in the supernatant of liquid cultures, and $A C P L$ is a putative pseudogene or serves a function as yet unidentified. The cloned genes will help clarify the contribution of fungal secreted proteases to virulence and can be used for molecular studies of $A C P$ expression following phagocytosis.

\section{Methods}

Strains and plasmids. Ten strains of $C$. parapsilosis were isolated from patients at the Centre Hospitalier Universitaire Vaudois (CHUV) and were maintained on Sabouraud agar medium. C. tropicalis ATCC 750 ade2/ade2, acp $\Delta:: \lambda / a c p \Delta:: \lambda$ (strain DSY59) is homozygous for the disrupted $A C P$ gene (Sanglard et al., 1992). The plasmid used to transform C. tropicalis DSY59, pMK16, was obtained from the Squibb Institute for Medical Research (Princeton, New Jersey, USA) and was described in Kurtz et al. (1987). C. albicans Ca74 is a clinical strain isolated at the CHUV (Togni et al., 1991). E. coli LE392 was used for the propagation of bacteriophage $\lambda$ EMBL3 (Promega). All plasmid subcloning experiments were performed in $E$. coli strain DH $5 \alpha$ using the plasmid pMTL21 (Chambers et al., 1988).

Liquid cultures. Yeast were grown at $30^{\circ} \mathrm{C}$ in Sabouraud liquid medium or, to promote production of $\mathrm{ACP}$, in $1.2 \%(\mathrm{w} / \mathrm{v})$ yeast carbon base (YCB) medium supplemented with $0.2 \%$ BSA and adjusted to $\mathrm{pH} 4.0$ with $1 \mathrm{M}-\mathrm{HCl}$. The latter medium was sterilized by filtration.

ACP production on solid medium. The medium contained $1.2 \%(\mathrm{w} / \mathrm{v})$ YCB, $0.2 \%$ BSA and $1.5 \%(w / v)$ agar. Nine hundred millilitres of a solution containing YCB and agarose was adjusted to $\mathrm{pH} 4.0$ with $1 \mathrm{M}-$ $\mathrm{HCl}$, and sterilized by autoclaving at $120^{\circ} \mathrm{C}$. BSA $(2 \cdot 0 \% \mathrm{w} / \mathrm{v}, 100 \mathrm{ml})$ was sterilized by filtration and added to cooled YCB-agarose to form a milky medium which was poured into Petri dishes. Cultures were incubated for $4 \mathrm{~d}$ at $30^{\circ} \mathrm{C}$ and ACP activity was observed as a clearing zone around the colonies. Agar plates were subsequently stained with amido black $0.1 \%$ in acetic acid/methanol/water (10:25:65, by vol.) and destained with $10 \%(\mathrm{v} / \mathrm{v})$ acetic acid to confirm proteolytic activity around the colonies.

Enzyme purification and $\mathrm{N}$-terminal amino acid sequence. Supernatants were separated from yeast cultures $(500 \mathrm{ml})$ by centrifugation at $5000 \mathrm{~g}$. Ammonium sulphate was dissolved in the supernatants at room temperature to $65 \%$ saturation. The precipitated proteins were collected by centrifugation and resuspended in distilled water at $1 / 100$ of the original volume. Insoluble material was removed by centrifugation at $5000 \mathrm{~g}$ for $5 \mathrm{~min}$ and the supernatant was dialysed against $15 \mathrm{~mm}$-sodium citrate ( $\mathrm{pH} \mathrm{56}$ ) for $2 \mathrm{~h}$. After reduction of the volume to $5 \mathrm{ml}$ by ultrafiltration (Ultracent-30 system, BioRad), the enzyme solution was chromatographed on a column of polyacrylamide P60 gel in 20 mm-sodium citrate buffer (pH 5.6). Fractions with enzymic activity were retained and pooled.

Protease activity was determined with azoalbumin as substrate as described (Sanglard et al., 1992). Protein concentration was measured by Lowry's method with BSA as the standard. SDS-PAGE was performed as described by Laemmli (1970), using $9 \%(\mathrm{w} / \mathrm{v})$ polyacrylamide gels. Gels were either stained with Coomassie Brilliant Blue R-250 or, for glycoproteins, with the periodic acid-Schiff reagent (PAS) (Zacharius et al., 1969). Determination of the N-terminal amino acid sequence of ACP was performed as described previously by JatonOgay et al. (1992).

Immunological methods. Preparation of anti-ACP immune sera and immunoblotting were performed as described by Monod et al. (1991).

Construction of the genomic library. A genomic DNA library was prepared using DNA from the $C$. parapsilosis isolate CHUV E18. Genomic DNA (Sanglard et al., 1992) was partially digested with Sau3A, and DNA fragments of $12-20 \mathrm{~kb}$ were isolated from low melting agarose (BioRad) (Sambrook et al., 1989). These fragments were inserted into bacteriophages using the $\lambda$ EMBL3 BamHI arm cloning system (Promega).

Screening of the genomic library. Approximatively 50000 recombinant plaques of the genomic library were immobilized on nylon membranes (Zeta-Probe, BioRad). The filters were hybridized with ${ }^{32} \mathrm{P}$ labelled C. tropicalis $A C P$ probes (Togni et al., 1991), in a solution containing $5 \times \mathrm{SSC}, 7 \%(\mathrm{w} / \mathrm{v}) \mathrm{SDS}, 10 \times$ Denhardt's, and $20 \mathrm{~mm}$ sodium phosphate $\left(\mathrm{pH} \mathrm{7.0)}\right.$ ), at $50^{\circ} \mathrm{C}$ for $24 \mathrm{~h}$. The membranes were exposed to X-ray film after a first wash in $3 \times$ SSC, $5 \%(w / v)$ SDS,

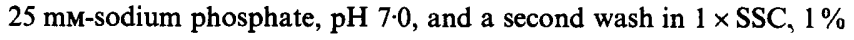
(w/v) SDS, at $50^{\circ} \mathrm{C}$. Positive plaques were purified and the DNA was isolated as described (Grossberger, 1987). Agarose gel electrophoresis of restricted recombinant bacteriophage $\lambda$ EMBL3 DNA and Southern blotting were performed according to standard protocols (Sambrook et al., 1989). DNA probes were labelled by random primer extension using a Boehringer kit and $\left[\alpha^{32} \mathrm{P}\right]$-dCTP (Amersham).

DNA sequencing. Double stranded DNA subcloned into plasmid pMTL 21 (Chambers et al., 1988) was sequenced using a Sequenase version 2.0 sequencing kit (USB) following the supplier's instructions. DNA was annealed with the reverse primer (Biofinex, Praroman), SL primer (USB) or synthetic oligonucleotide primers (Microsynth, Windisch).

DNA transformation. E. coli was transformed using competent cells with standard protocols (Sambrook et al., 1989). C. tropicalis was transformed by protoplasting as described by Sanglard et al. (1992).

Protein comparisons. Identity and similarity scores (\%) between Candida acid proteases, were calculated using the algorithm of Needleman \& Wunsch (1970) implemented in the GCG Wisconsin program.

\section{Results}

Purification and properties of the C. parapsilosis $A C P$

All 10 strains of $C$. parapsilosis examined exhibited comparable proteolytic activities on BSA agar plates, 


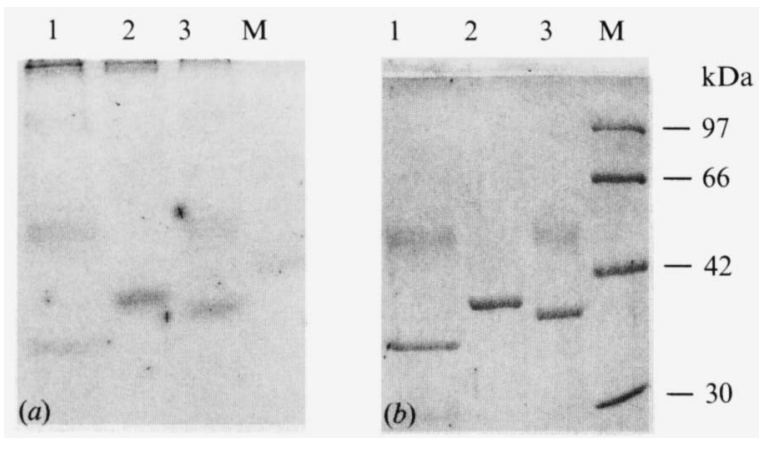

Fig. 1. (a) PAS positive staining of $C$. parapsilosis E18 (lane 1), C. tropicalis ATCC 750 (lane 2) and C. albicans Ca74 (lane 3) ACP. (b) As $(a)$ but the gel was stained with Coomassie Brillant Blue R-250. Proteins were precipitated from the culture supernatant with $65 \%$ saturated ammonium sulphate and separated by SDS-PAGE ( $9 \%$ gels, w/v). M, molecular mass markers: phosphorylase $\mathrm{B}, 97.4 \mathrm{kDa}$; bovine serum albumin, $66.2 \mathrm{kDa}$; ovalbumin, $42.7 \mathrm{kDa}$; bovine carbonic anhydrase, $31 \mathrm{kDa}$.

thus, protease purification and characterization was performed only with one strain (CHUV E18).

In a typical experiment, a total of $15000 \mathrm{U}$ of proteolytic activity was produced in one litre of BSA medium after $4 \mathrm{~d}$ growth at $30^{\circ} \mathrm{C}$. The $C$. parapsilosis ACP was purified as described in Methods, with a yield of $50 \%$. A single peak of proteolytic activity was obtained after P60 gel chromatography that corresponded to the major peak from the gel column. The purified enzyme showed a single protein band in SDSPAGE gel at $37 \mathrm{kDa}$, corresponding to the molecular mass of the protease obtained by P60 gel chromatography. Staining with PAS reagent indicated that this enzyme was a glycoprotein (Fig. $1 a$ ). The enzyme was a major protein secreted by the fungus as shown by the protein profile of the $65 \%$ saturated ammonium sulphate precipitate of the culture supernatant. The initial 15 amino acid residues of the $\mathrm{N}$-terminus were determined to be Asp-Ser-Ile-Ser-Leu-Ser-Leu-Ile-Asn-GluGly-Pro-Ser-Tyr-Ala. The ACP of C. parapsilosis was related to ACP of $C$. albicans and $C$. tropicalis. Using a polyclonal antibody raised against the $C$. parapsilosis ACP, crossreactivities with the ACP from $C$. albicans and $C$. tropicalis could be observed in immunoblotting experiments (data not shown).

\section{Cloning and sequencing of the putative $C$. parapsilosis ACP gene}

A $2 \mathrm{~kb} B g / \mathrm{II}-E c o$ RI fragment, containing the entire $C$. tropicalis $A C P$ gene (Togni et al., 1991) was used as the probe to screen the $C$. parapsilosis $\lambda \mathrm{EMBL} 3$ genomic library. Four hybridizing clones were identified. Restriction enzyme digestion of purified DNA revealed that these clones carried similar but not identical DNA sequences. However, all four clones contained a $7.6 \mathrm{~kb}$ HindIII fragment which hybridized to the probe (data not shown). This fragment, for which a restriction map is shown in Fig. 2, was subcloned in pMTL 21 (Chambers et al., 1988), generating the plasmid pMTL 21-H7. Further analysis revealed that the $7.6 \mathrm{~kb}$ HindIII fragment contained two distinct segments that hybridized with the probe, separated by a $3 \mathrm{~kb}$ intervening sequence. Nucleotide sequencing of the two segments revealed two long ORFs of 1206 and $1185 \mathrm{bp}$, with opposite directions of transcription. The genes for these two ORFs were named $A C P R$ and $A C P L(\mathrm{R}$ and $\mathrm{L}$ are relative to their right and left positions on Fig. 2). Their sequences, including the $5^{\prime}$ and $3^{\prime}$ flanking regions, are shown in Fig. 3. Both ORFs were preceded at $-81(A C P R)$ and -70 $(A C P L)$ bp (relative to the first ATG codon) by a TATAAAT sequence which constitutes a possible TATA box (Corden et al., 1980).

\section{Amino acid sequences deduced from $A C P R$ and $A C P L$}

The amino acid sequences deduced from $A C P R$ and $A C P L$ showed similarity with other proteases, in particular within three regions which are highly conserved in all members of the pepsin family. Two of these regions contained the two reactive aspartic acid residues of the active site for these enzymes. Both $C$. parapsilosis ORFs suggested the existence of a signal peptide with putative signal peptidase cleavage sites (von Heijne, 1986), indicated by arrows on Fig. 3. Furthermore, pairs of Lys-Arg residues which are known to be proteolytic processing sites in other yeast secretion signal sequences (Julius et al., 1984; Davidow et al., 1987) were found at about position 30 and 60 relative to the Met 1 residue. These sites are also conserved in $C$. albicans and $C$. tropicalis ACP prosequences (Hube et al., 1991; Togni et

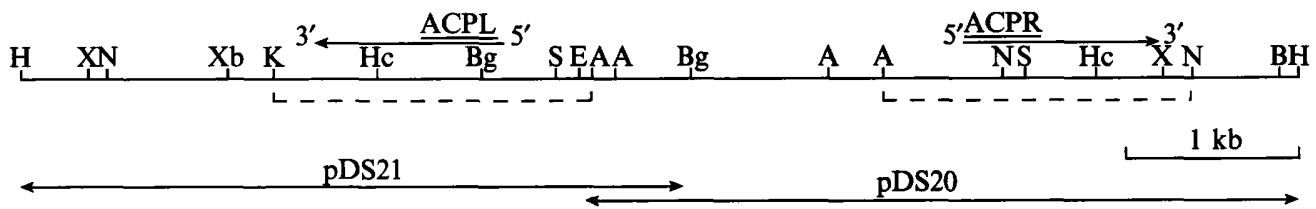

Fig. 2. Restriction map of the $7.6 \mathrm{~kb}$ HindIII fragment obtained from positive recombinant bacteriophages. The two ORFs described in this work are represented by horizontal arrows. The two segments for which the sequence are reported in this work are dotted. The fragments inserted in pMK16 to generate pDS20 and pDS21 (Sanglard et al., 1992) are indicated below the map. Symbols: A, AccI; B, BamHI; Bg, BglII; E, EcoRI; H, HindIII; Hc, HincII; K, Kpn; N, NsiI ; S, SacI; X, XhoI; Xb, XbaI. 


\section{ACPR}

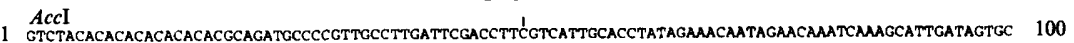

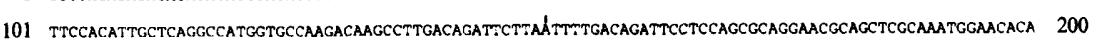
201 AAATCAAAAAATGCACTTCCCACACTTTCCCGTCCCACGGCTCTAATTGTĆTGTTTCAGGTTTACAATTTGAACTGGCAATGATAAGACTGCTGAAGTGA 300 301 TACAAATCACAAGACAATGGATTCACGGGCTGTGGCTTAAGCCGCCAGATATACCAGATAATGACTAAACGCAATCAGCTTCGTGCCTCATGGGCTCGTA 400 401 COGCACCAAAGCTGACAAACACCAATTACAAATTCAGTCAA FATAAA ACCCCATGTGCCTTCTCTTTTTCATCCACTGGTCATTCCCATCCATTGCCAA 500 501 ATAAAAACGCTATCCATACAAATGGTTGCCATTCTTACCTTAACTCGTCAAGTTTTACTCACCATCGCGCTCGCTTTGTTTCCACAAGGTGCAGCTATT 600 601 CCAGAGGAGGCTGCTAAAAGAGACGACAATCCTGGGTTTCTTGCCTTGGACTTTCATGTGCTTAGGAAACCATTGAACTTGACCGAGGCGCTTCTCCCTG 700 $P E$ E A A

701 AAAAGAGACACTCCATTTCGTTGTCGTTGATCAATGAaGGTCCATCATATGCATCTAAAGTTTCAGTCGGTTCAAACAAACAGCAGCAAACCGTCATTAT 800

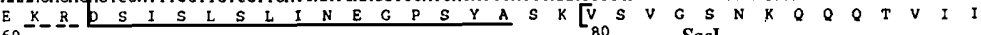

801 TGATACTGGTTCGAGTGACTTTTGGGTAGTGGATTCAAATGCCCAATCTGGAAAAGGTGTTGATTGCAAGAGCTCAGGGACCTTTACCCCATCATCGTCT 900

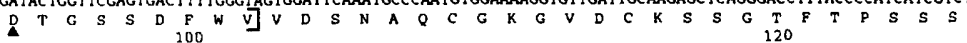

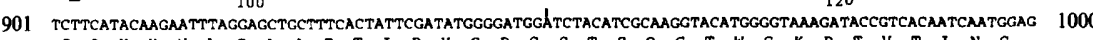
S S Y K N L G A A F T T R Y G

1001 TCTCAATCACTGGACAACAAATTGCAGATGTCACTCAAACATCAGTTGATĆAAGGTATATTGGGTATTGGCTACACCAGCAATGAaGCAGTTTACGACAC 1100

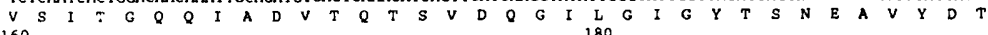

101 GAGTGGTCGTCAAACCACTCCAAACTACGACAACGTTCCCGTGACTTTGAhAAAGCMAGGAAGGATCAGAACCAATGCTTACTCATTATATTTGAACTCT 1200

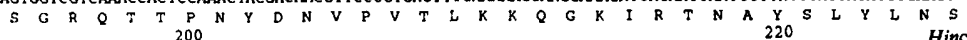

1201 CCTTCAGCTGAGACTGGTACAATCATCTTTGGTGGTGTTGATAATGCCAAGTACTCTGGCAAGTTGGTTGCCGAGCAAGTTACCCTGTCACAGCCGTTGA 1300

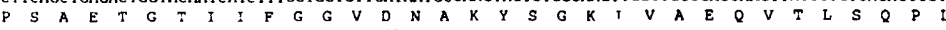

1301 СTATT:CGCTTGCTTCCGTCAATTTCAMGGTTCATCATTTTCATTTGGAGATGGTGCTTTGTTC, A.TCTGGAACGACACTCACTTACTTCCCAAGTGA 1400

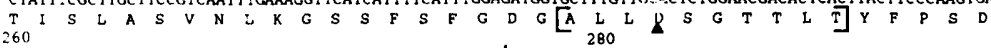

1401 CT:TGCCGCACAACTTGCTGATAAACCTGGTGCTCGTCTTGTTCAAGTGGĆTAGAGACCAATACTTGTACTTTATTGATTGTAACACCGATACATCTGGC 1500

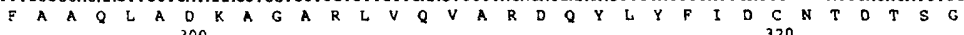

501 ACAACGGTGTTTAACTTTGGAATCGGGCAAGATTACCGTTCCAAACACÁ́GAGTACGTTTATCAAAATGGTGATGGTACTTGTCTTTGGGGTATCCAAC 1600

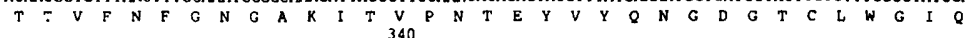

1601 CATCAGACGATACTATTTTAGGTGACAACTTTTTGAGACATGCTTATTAC'CTCCTTTACAACTTGGATGCAAACACAATCTCAATCGC:TCAGTCAAGTA 1700

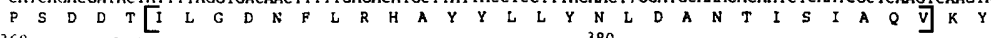

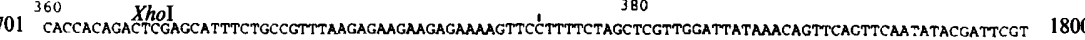
$T$ T D S S S I S A V

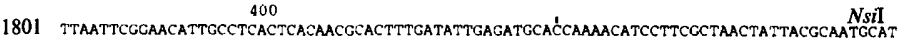

1885

\section{ACPL}

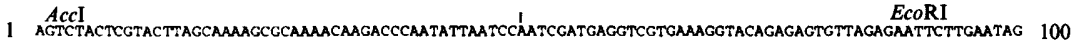
101 ACACATCTACCAGTGGGCGCTGGGCTGTTTTTGGCACAGGTGGGTAGCAACCATTTCAAATTACCTTGAACCACGTJGTTTGTGCTTGTTTTACAACTT 200 201 CCTCGGAGGAGCTCCTTCCATTCTCATAACCACTTCTTTGGCTGAaTTT'́GGCCCATGCGCATATCCAT:TGAaTAaTTGAGCCAATCAAGTGAaCAACC 300 301 GAAGAGAGTGGAAAGAAATGCCTTTGCAGGAGTCAATTATGGCAGTCAAÁCTCCTTTCTGCCAGAGTCTCATTGGCCCACGTTGGTGTTTACACCGGTGC 400 401 CCTTGGAACAT ATAAA AGGGAGGAAATCCCCAAGATTGGGTGAGTTAATGTCAAGTCACCAMCTATCAATTACTCCAATGACTACAATTGCCATC 500

501 TTTACTAAAACGTTTTGCTTGCCATTGCTTTTGCTTTGTTTGCCCAAGGTGCTGCTATTCCAGACCCCGCTAAAaGAGATGATAACCCTGCTT:TGTTC 600 F T T K N

601 CTTTGGATTTTGAaGTTACAAGAAAGCCTCTCGATGTGAATGCTACTTCAGAACTTTCTAMAGGATCTTCTCCATCATCACCATTGTACT:TGAGGGACC 700

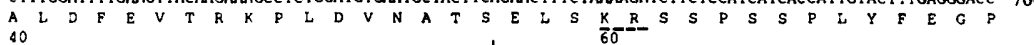

701 TTCATATGGTATTCGTGTTTCAGTTGGTTCCAACAAACAAGAACAACAAGTGGTTCTTGACACCGGTTCTAGTGACTTTTGGGTCGTTGATTCCTCTGCa 800

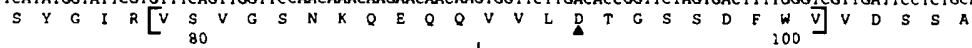

801 TCTTGTCAAAAGGTAATTGTAAGCATATGGAACTTTTGACCCACATAGCTCAACCTCATTCAAGTCATTAGGCTCCAGTTTCAGCATTCGTTATGGGG 900 S C Q K G N C K Q Y Y G T F D P H S S T S F K S L G S S F S I G Y G

901 ACAAGAGTTCTTCGATTGGAACTTGGGGGCAAGATACAATTTATCTTGG'GGTACTTCCATTACCAATCAACGCTTTGCTGATGTTACCAGCACCTCGGT 1000 $\begin{array}{lllllllllllllllllllllllllllllllllll}D & K & S & S & S & I & G & T & W & G & Q & D & T & I & Y & L & G & G & T & S & I & T & N & Q & R & F & A & D & V & T & S & T & S & V\end{array}$

1001 CAATCAACGAATATTAGGTGTTGGACGTGTGGAAACTGAGAGTCCAAATCCCCCATATCACAMCGTCCCTATCACTTTCAAAAAGCAAGGAAAAATCAAG 1100 N Q G I L G G G R V E

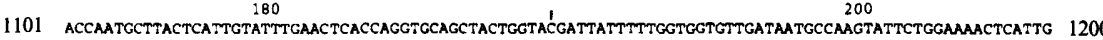
T N N A

1201 AGGAGCCATTGGTTC:GGACCGATACTTGGCTGTAAACTTGAAATCCCTT'AACTACAATGGCGATAACTCCAACGCGGGTTTTGGTGTTGTTGTGGATTC 1300 E E P L V L D R Y L A V N L K S L L N Y N G D N S N A G F G E V V D 1301240 CGGAACCACAATTAGTTACTTGCCTGACAGCATCGTTAACGATCTCGCCAACAAGGTTGGTGCATACTTGGACCCAGTTGGTTTGGGAAACGAGCTTTAC 1400 G $T$ T $T$ T I

201 TTTATTGATTGTAATGCCAATCCTCAAGGTAGTGCTTCTTTCACCTTCGÁCAATGGTGCTAAGATTACTGTTCCATTATCTGAATTTGTCCTTCAAAGTA 1500 F I I D C C N A A N

1501 CTGCCAATGCTTCCGTCTGGGGTTTACAAAGTTCCGATAGACAMATGTTCCTCCAATCTTGGGTGATAATTTTTTGAGACACGCTTATGTCGTTTTCAA 1600

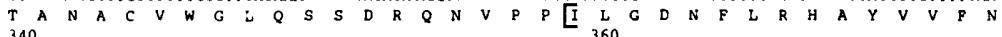

1601 T:TGGATAAAGAGACCGTTTCTCTCGCTCAGGTGAAGTACACTTCTGCCTCAAGTGTTTCAGCAATTTAGAAACTTCCATTGT TTGGAAACCTTTTTT: 1700

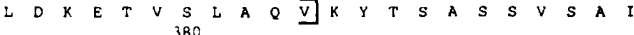

1701 CAATCGTTATATTCAATAAAT:GTGTTGTTATGAGACAAATCTGTAGTGTAATTTCTGGCAATTGTTGTGCATCAAGAGCGGCGATAAGGAaCAAATTSS 1800

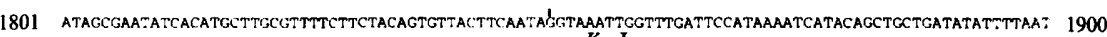

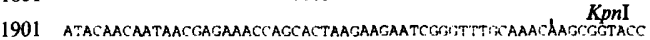

Fig. 3. Nucleotide sequence of $C$. parapsilosis $A C P R$ and $A C P L$. The deduced amino acid sequence is shown below the nucleotide sequence. The N-terminal amino acid sequence of the mature C.parapsilosis ACP begins at residue 63 in $A C P R$ (horizontal arrow). The 15 amino acid sequence from the $\mathrm{N}$-terminus of the protein determined by amino acid sequencing is underlined. The two Lys-Arg peptidase processing sites found in the $A C P R$ propeptide sequence and the homologous sites in $A C P L$ are dotted. Putative cleavage sites within the signal sequence are indicated by vertical arrows. Aspartic acid residues homologous to those of the active site of pepsin-like proteins are marked by a filled triangle. Three domains showing strong homology with proteins which are members of the pepsin family are delineated with brackets. Possible TATA sequences are boxed. 


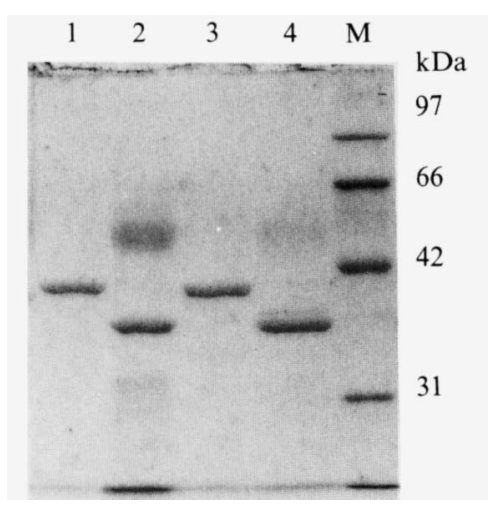

Fig. 4. Protein profiles of culture media of Candida tropicalis ATCC 750 (lane 1), C. parapsilosis E18 (lane 2), C. tropicalis acp mutant complemented by $C$. tropicalis $A C P$ (lane 3 ) and by $A C P R$ (lane 4). The proteins precipitated from the culture medium with $65 \%$ saturated ammonium sulphate were visualized by $9 \%(w / v)$ SDS-PAGE. The gel was stained with Coomasie Brilliant Blue R-250. Molecular mass markers as used in Fig. 1.

al., 1991). The $\mathrm{N}$-terminal amino acid sequence of mature C. parapsilosis ACP, obtained by Edman amino acid sequencing of the purified enzyme, could be found in the $A C P R$ deduced amino acid sequence only. It was preceded by the second Lys-Arg tandem sequence, as previously found for ACP of C. tropicalis and C. albicans. In $A C P L$ the amino acid sequence following the second Lys-Arg residue was different from that of $A C P R$, and no sequence identical to the $\mathrm{N}$-terminus of the $A C P R$ product could be found in the $A C P L$ sequence. The amino acid sequencing of the $\mathrm{N}$-terminus of $C$. parapsilosis ACP prepared after precipitation of the proteins from liquid culture supernatants with $100 \%$ saturation ammonium sulphate did not identify another exoprotease. These findings suggest that only the $A C P R$ product was secreted in the medium in the presence of BSA as a nitrogen source.

Complementation of a C. tropicalis acp mutant with acid protease genes from $C$. parapsilosis

Both the $A C P R$ and $A C P L$ sequences were subcloned into pMK16, a plasmid able to replicate in a ade $2 C$. tropicalis mutant (Sanglard et al., 1992). The recombinant plasmids pDS20 and pDS2 1 carrying $A C P R$ and $A C P L$, respectively (Sanglard et al., 1992), were used to transform C. tropicalis DSY59, a strain homozygous for the disrupted $A C P$. A clearing zone on BSA-agar was obtained only for the yeasts transformed with pDS20. The expressed protease was isolated from the supernatant of a BSA liquid culture of these transformants and showed the same mobility on SDS-PAGE as ACP secreted by $C$. parapsilosis (Fig. 4). The C. tropicalis acp mutant transformed with pDS21 did not grow in BSA liquid medium.

\section{Discussion}

We report here the purification of $C$. parapsilosis ACP secreted by the yeast when BSA is the sole nitrogen source and the cloning, sequencing and functional characterization of the corresponding gene. The enzyme we isolated from C. parapsilosis E18 is similar to that characterized by Rüchel (1986) in having a lower molecular mass than C. albicans and C. tropicalis ACP. The ACP isolated from other $C$. parapsilosis strains showed identical molecular mass to that of strain E18 (data not shown).

Evidence for the cloning of the C. parapsilosis $A C P$ gene included the following. (i) The gene we identified as $A C P R$ contained a DNA sequence corresponding to the $\mathrm{N}$-terminal 15 aa sequence of purified $C$. parapsilosis ACP protein. (ii) When $A C P R$ was expressed in a $C$. tropicalis acp mutant, ACP activity was restored, and (iii) the protease secreted from the transformed acp mutant showed the same mobility on SDS-PAGE as the protease secreted by wild type $C$. parapsilosis.

Inspection of the deduced $\mathrm{N}$-terminal amino acid ACPL sequence beginning from the Met 1 residue suggests the existence of a precursor sequence similar to that of $C$. albicans and C. tropicalis ACP, and ACPR product. This precursor sequence has a secretion signal sequence of 14-21 amino acids with 4 putative signal peptidase cleavage sites (von Heijne, 1986) and two tandem Lys-Arg sequences which are proteolytic processing sites in C. tropicalis and C. albicans ACP (Togni et al., 1991; Hube et al., 1991), and also in the $A C P R$ product. The presence of $A C P L$ secretion signal sequence similar to those of other Candida ACP seems to indicate that $A C P L$ has no defect in secretion capability. However, only one acid protease, the $A C P R$ product, was found in supernatants of liquid cultures of $C$. parapsilosis, and ACPL could not be expressed in the $C$. tropicalis acp mutant. $A C P L$ could be considered as a pseudogene, but it can not be excluded that $A C P L$ may be expressed under not identified conditions. Indeed an acid protease gene of $C$. albicans WO-1, Opla ( $99 \%$ homologous to $C$. albicans $A C P 1$ on Fig. 5), is transcribed only when the cells switch from a white to opaque form (Morrow et al., 1992). This feature is strain dependent: in C. albicans 3153A this gene remains silent, although present in the genome. When, however, BSA is added to the culture medium, the stimulation of extracellular proteolytic activity in $C$. albicans is due to the expression of another acid protease gene functionally analogous to $A C P R$. Recent studies showed that $C$. albicans, like $C$. parapsilosis, contains at least two genes encoding secreted acid proteases (Wright et al., 1992).

Immunoblotting experiments with an anti-ACP polyclonal antibody suggested that $C$. tropicalis, $C$. albicans 


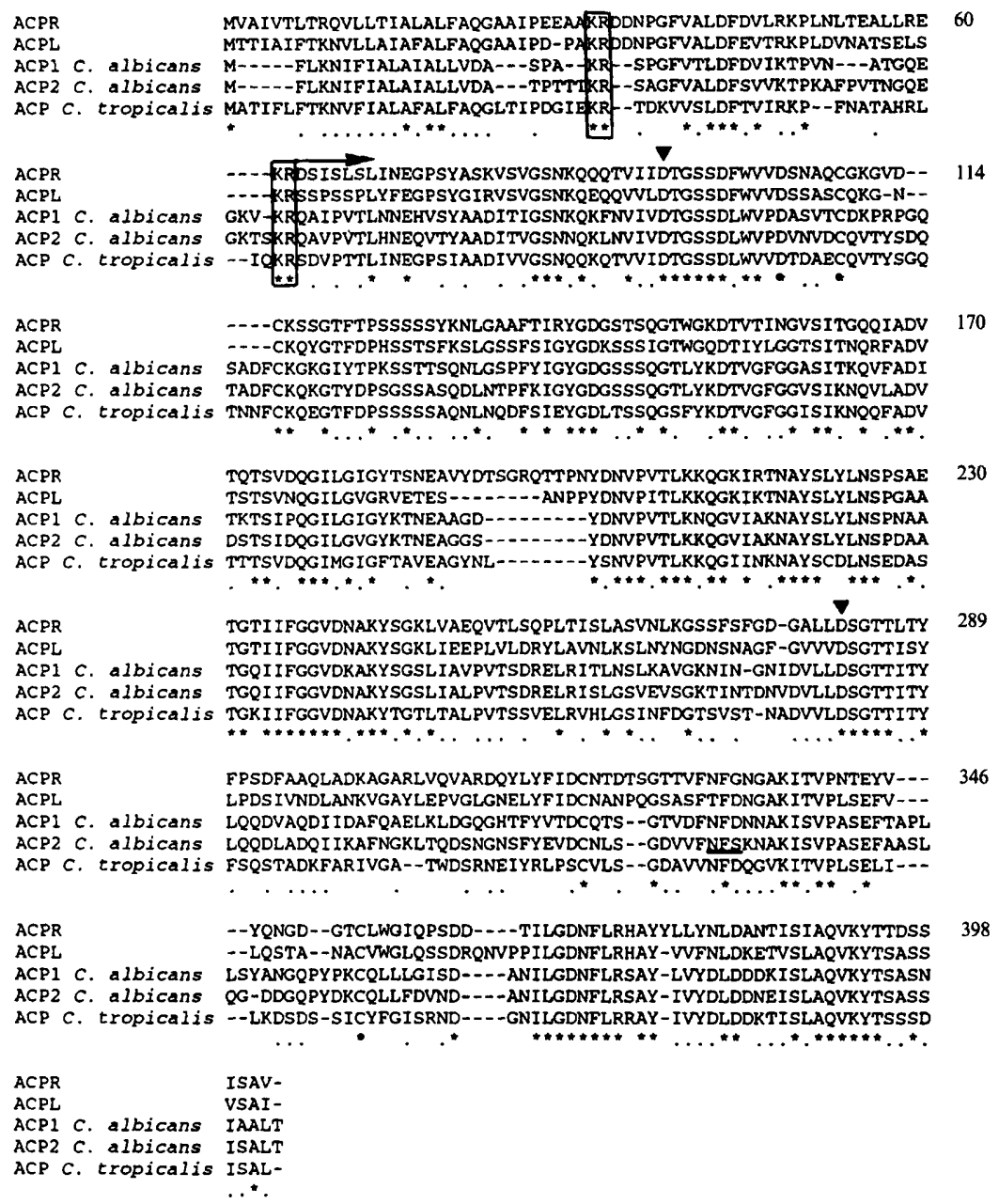

Fig. 5. Comparison of acid protease amino acid sequences from C. parapsilosis $A C P R$ and $A C P L, C$. albicans and C. tropicalis $A C P$. The numbering is that of the $C$. parapsilosis $A C P R$ product. Filled triangles identify aspartic acid residues corresponding to those found in the active site of proteases of the pepsin family. The $\mathrm{N}$-terminus of the mature secreted protein is indicated by a horizontal arrow. Tandem Lys-Arg sequences known to be proteolytic processing sites are boxed. A potential $N$-linked glycosylation site sequence $\mathrm{N}$ $\mathrm{F}-\mathrm{S}$ in $C$. albicans ACP2 is underlined. Asterisks and points show identical and similar amino acids, respectively. $A C P R, A C P L, C$. tropicalis ACP (Togni et al., 1991), C. albicans ACP1 (Hube et al., 1991) and ACP2 (Wright et al., unpublished) are available in the EMBL database with the accession numbers Z11919, Z11918, X61438, X56867 and M83663, respectively.

and C. parapsilosis ACP were closely related. However, the comparisons of the deduced amino acid sequences of the mature proteins show that they are far from being identical (Fig. 5). When ACP of two different species were compared, identity and similarity scores varied from 47.9 to $58.9 \%$ and from 62.2 to $70.1 \%$, respectively. High identities were found in the two domains containing the two aspartate residues of the pepsin family active site, and in a conserved domain at the C-terminal part of the proteins (Fig. 5). The theoretical pI of $C$. parapsilosis ACP is $4 \cdot 4$. This value is slightly, but notably, higher than those of $C$. albicans ACP2 (4.0) and C. tropicalis ACP (4-1).

The polypeptide chains of mature $C$. tropicalis, $C$. albicans and $C$. parapsilosis acid proteases have calcu- lated molecular masses of $35 \cdot 7,36 \cdot 3$ and $36 \cdot 0 \mathrm{kDa}$, respectively. The size of $C$. parapsilosis ACP, estimated from electrophoretic mobility on SDS-PAGE, is $4 \mathrm{kDa}$ smaller than that of $C$. tropicalis and C. albicans ACP. This discrepancy may be explained by the fact that the mature ACPs are glycoproteins, as shown by positive staining with PAS (Fig. $1 a$ ). The $C$. albicans and $C$. tropicalis ACPs were shown to be mannose proteins by MacDonald \& Odds (1980) and Rüchel et al. (1986), but glycosylation sites on the mature protein have not been investigated. Preliminary experiments on the expression of the C. tropicalis $A C P$ in the Saccharomyces cerevisiae phosphomannomutase deficient mutant sec53 (Kepes \& Schekman, 1988) confirmed the presence of mannose, since the expressed protein showed a smaller size than 
the native enzyme (data not shown). Inspection of the amino acid sequence of the mature protein reveals only one putative $\mathrm{N}$-glycosylation site present in a C. albicans $\mathrm{ACP}$ at position 321 (Fig. 5). Consequently, the $C$. parapsilosis ACP, like the other Candida ACPs must contain $O$-glycosylated residues and not $N$-glycosylated ones. However, glycosylation of Candida ACP remains to be further investigated biochemically.

Aspartic proteases are found in retroviruses and in eukaryotic cells, but not in bacteria. Retroviral aspartic proteases are dimers of identical subunits of 95-125 aa, each containing an active aspartic residue (Rao et al., 1991). Eukaryotic aspartic proteases consist of a polypeptide chain of about 320 aa which contains two active aspartic acid residues, one each in an $\mathrm{N}$ and $\mathrm{C}$-terminal domain. These two domains have only limited sequence homology except in the vicinity of their active site (Tang \& Wong, 1987). It is suggested that eukaryotic aspartic proteases evolved by duplication of an ancestral gene. Retroviral and eukaryotic aspartic proteases show a similar bilobal tertiary structure (Rao et al., 1991). The two lobes, identical in the case of retroviral aspartic proteases, are different in eukaryotic proteases, and correspond to the $\mathrm{N}$ and $\mathrm{C}$-terminal domains. These two lobes are separated by a deep cleft which contain the two aspartic residues of the active site. The three different Candida ACPs structurally resemble other eukaryotic aspartic proteases described. They have a polypeptide chain of about 340 aa residues and the locations of two conserved regions containing the two reactive aspartic acid residues are similar. Mammalian and plant aspartic proteases have three disulphide bridges in similar positions (Tang \& Wong, 1987; Runeberg-Roos et al., 1991). Like other fungal aspartic proteases (Horiuchi et al., 1988; MacKay et al., 1988) two disulphide bridges are apparently conserved in the three Candida secreted enzymes as attested by the four cysteine residues conserved in all acid proteases (Fig. 5).

Now that the genes encoding Candida ACP are available, experiments are planned to study the expression of these genes in macrophages. Further studies may show how differences in ACP translate to differences in virulence.

We thank Dr D. Ray and Dr M. Briehl for helpful discussions and assistance with the English, and A. Micolis and J. Chevalley for expert secretarial assistance.

\section{References}

Bistoni, F., Vecchiarelli, A., Cenci E., Sbaraglia, G., Perito, S. \& CASSONE, A. (1984). A comparison of experimental pathogenicity of Candida species in cyclophosphamide-immunodepressed mice. Sabouraudia 22, 409-418.

BORG, M. \& RüCHEL, R. (1990). Demonstration of fungal proteinase during phagocytosis of Candida albicans and Candida tropicalis. Journal of Medical and Veterinary Mycology 28, 3-14.

Chambers, S. P., Prior, S. E., Barstow, D. A. \& Milton, N. P. (1988). The pMTL nic-cloning vectors. 1. Improved polylinker region to facilitate the use of sonicated DNA for nucleotide sequencing. Gene 68, 139-149.

Corden, J., Wasylyk, B., Buchwalder, A., Sassone-Corsi, P., Kedinger, C. \& Chambon, P. (1980). Promoter sequences of eukaryotic protein-coding genes. Science 209, 1406-1414.

Davidow, L. S., O'Donnell, M. M., Kaczmarek, F. S., Pereira, D. A., Dezeeuw, J. R. \& Franke A. E. (1987). Cloning and sequencing of the alkaline extracellular protease gene of Yarrowia lipolytica. Journal of Bacteriology 169, 4621-4629.

GrossBerger, D. (1987). Minipreps of DNA from bacteriophage lambda. Nucleic Acids Research 15, 6737.

VON HEIJNE, G. (1986). A new method for predicting signal sequence cleavage sites. Nucleic Acids Research 14, 4683-4690.

Horiuchi, H., Yanai, K., Okazaki, T., Takagi, M. \& Yano, K. (1988). Isolation and sequencing of a genomic clone encoding aspartic proteinase of Rhizopus niveus. Journal of Bacteriology 170, 272-278.

HoRN, R., Wong, B. \& KieHN, T. E. (1985). Fungemia in a cancer hospital: changing frequency, earlier onset, and results of therapy. Reviews of Infectious Diseases 7, 646-654.

Hube, B., TURVER, C. J., OdDS, F. C., EIFFerT, H., BOUlnoIs, G. J., KOCHEL, H. \& RüCHEL, R. (1991). Sequence of the Candida albicans gene encoding the secretory aspartate proteinase. Journal of Medical and Veterinary Mycology 29, 129-132.

Jaton-Ogay, K., Suter, M., Crameri, R., Falchetro, R., Fatih, A \& MoNOD, M. (1992). Nucleotide sequence of a genomic and a cDNA clone encoding an extracellular alkaline protease of $\mathrm{As}$ pergillus fumigatus. FEMS Microbiology Letters 92, 163-168.

Julius, D., BRAKe, A., BlaiR, L., KunisAWA, R. \& ThORNER, J. (1984). Isolation of the putative structural gene for the lysine-argininecleaving endopeptidase required for processing of yeast prepro- $\alpha$ factor. Cell 37, 1075-1089.

KePES, F. \& SChEKMAN, R. (1988). The yeast SEC53 gene encodes phosphomannomutase. Journal of Biological Chemistry 263, 91559161.

Komshian, S. V., Uwaydah, A. K., Sobel, J. D. \& Crane, L. R. (1989). Fungemia caused by Candida species and Torulopsis glabrata in the hospitalized patient: frequency, characteristics, and evaluation of factors influencing outcome. Reviews of Infectious Diseases 11, 379-390.

KurtZ, M. B., Cortelyou, M. W., Miller, S. M., Lai, M. \& Kirsch, D. R. (1987). Development of autonomously replicating plasmids for Candida albicans. Molecular and Cellular Biology 7, 209-217.

Kwon-Chung, K. J., Lehman, D., Good, C. \& Magee, P. T. (1985). Genetic evidence for role of extracellular proteinase in virulence of Candida albicans. Infection and Immunity 49, 571-575.

LAEMMLI, U. K. (1970). Cleavage of structural proteins during the assembly of the head of bacteriophage T4. Nature, London 227, 680-685.

MACDonald, F. \& ODDS, F. C. (1980). Inducible proteinase of Candida albicans in diagnostic serology and in the pathogenesis of systemic candidosis. Journal of Medical Microbiology 13, 423-435.

MacDonald, F. \& OdDs, F. C. (1983). Virulence for mice of a proteinase secreting strain of Candida albicans and a proteinasedeficient mutant. Journal of General Microbiology 129, 431-438.

MacKay, V. L., Welch, S. K., InSley, M. Y., Manney, T. R., Holly, J., SAARI, G. C. \& PARKeR, M. L. (1988). The Saccharomyces cerevisiae $B A R 1$ gene encodes an exported protein with homology to pepsin. Proceedings of the National Academy of Sciences of the United States of America 85, 55-59.

Monod, M., ToGNi, G., Rahalison, L. \& FrENK, E. (1991). Isolation and characterisation of an extracellular alkaline protease of Aspergillus fumigatus. Journal of Medical Microbiology 35, 23-28.

MORROW, B., SRIKANTHA, T. \& Soll, D. R. (1992). Transcription of the gene for a pepsinogen, $P E P 1$, is regulated by white-opaque switching in Candida albicans. Molecular and Cellular Biology 12, 2997-3005.

Needleman, S. B. \& Wunsch C. D. (1970). A general method 
applicable to the search for similarities in the amino acid sequence of txo proteins. Journal of Molecular Biology 48, 443-453.

Negi, M., TsuboI, R., MatsuI, T. \& OgaWA, H. (1984). Isolation and characterization of proteinase from Candida albicans: substrate specificity. Journal of Investigative Dermatology 83, 32-36.

RaO, J. K. M., ERICKSON, J. W. \& Wlodawer, A. (1991). Structural and evolutionary relationships between retroviral and eucaryotic aspartic proteinases. Biochemistry 30, 4663-4671.

RAY, T. \& PAYNE, C. D. (1990). Comparative production and rapid purification of Candida acid proteinase from protein-supplemented cultures. Infection and Immunity 58, 508-514.

Remold, H., Fasolk, H. \& Staib, F. (1968). Purification and characterization of a proteolytic enzyme from Candida albicans. Biochimica et Biophysica Acta 167, 399-406.

Ross, I. K., De Bernardis, F., Emerson, G. W., Cassone, A. \& Sullivan, P. A. (1990). The secreted aspartate proteinase of Candida albicans: physiology of secretion and virulence of a proteinasedeficient mutant. Journal of General Microbiology 136, 687-694.

RüCHEL, R. (1981). Properties of a purified proteinase from the yeast Candida albicans. Biochimica et Biophysica Acta 659, 99-113.

RüChel, R. UhlemanN, K. \& BöNING, B. (1983). Secretion of acid proteinases by different species of the genus Candida. Zentralblatt für Bakteriologie Mikrobiologie und Hygiene A 255, 537-548.

RÜCHEL, R., BONING, B. \& BoRG, M. (1986). Characterization of a secretory proteinase of Candida parapsilosis and evidence for the absence of the enzyme during infection in vitro. Infection and Immunity 53, 411-419.
RÜCHel, R., ZimmermanN, F., BöNING-STUTZER, B. \& HeLmChEN, U. (1991) Candidiasis visualised by proteinase-directed immunofluorescence. Virchows Archiv A Pathological Anatomy and Histopathology 419, 199-202.

RuNeBerg-Roos, P., TörmäKangas, K. \& Östman, A. (1991). Primary structure of a barley-grain aspartic proteinase. European Journal of Biochemistry 202, 1021 1027.

Sambrook, J., Fritsch, E. F. \& Maniatis, T. (1989). Molecular Cloning: a Laboratory Manual, 2nd edn., Cold Spring Harbor, NY : Cold Spring Harbor Laboratory Press.

Sanglard, D., Togni, G., DE Viragh, P. A. \& Monod, M. (1992). Disruption of the gene encoding the secreted acid protease $(A C P)$ in the yeast $C$. tropicalis. FEMS Microbiology Letters 95, 149-156.

Shimizu, K., KondoH, Y. \& TANaKa, K. (1987). Proteinase production and pathogenicity of Candida albicans. Microbiology and Immunology 31, 1045-1060.

TANG, J. \& WoNG R. N. S. (1987). Evolution in the structure and function of aspartic proteases. Journal of Cellular Biochemistry 33, 53-63.

Togni, G., Sanglard, D., Falchetto, R. \& Monod, M. (1991). Isolation and nucleotide sequence of the extracellular acid protease gene $(A C P)$ from the yeast Candida tropicalis. FEBS Letters 286, 181-185.

Wright, R. J., Lamont, I. L., Emerson, G. W. \& Sullivan, P. A. (1992). The aspartyl proteinase of Candida albicans. Yeast 8, S662.

Zacharius, R. M., Zell, T. E., Morrison, J. H. \& Woodlock, J. J. (1969). Glycoprotein staining following electrophoresis on acrylamide gels. Analytical Biochemistry 30, 148-152. 